Aus der Kgl. Universitätsklinik für Hautkrankheiten zu Breslau. (Geheimrath Prof. Dr. Neisser.)

\title{
Ueber die Action des Quecksilbers auf das syphilitische Gewebe.
}

\author{
Von \\ Dr. Pollio, \\ Volontär-Assistent an der Klinik.
}

Die Arbeit von Justus: „Die Action des Quecksilbers auf das syphilitische Gewebe", welche im LVII. Bd. dieses Archirs publicirt ist, musste unser Interesse in doppelter Hinsicht erwecken, nicht allein, weil die von $J$ u s $t u$ s angegebene Methode den unmittelbaren Nachweis von Quecksilber im Gewebe gestatten sollte, sondern auch weil die Methode uns in der Erkenntniss des Verhaltens des Quecksilbers gegenüber dem syphilitischen Gewebe zu fördern, d. h. das Wesen der specifischen Therapie zu ergründen versprach.

Ich leistete daher gern der Anregung meines hochverehrten Chefs, des Herrn Geheimrath $\mathrm{N}$ e is s e r, dem ich auch an dieser Stelle bestens danke, Folge, diese interessanten Befunde mir selbst durch das von $\mathrm{Justus}$ angegebene Verfahren darzustellen.

Ich begann nun, genau nach $J u$ s $t$ u s' Angaben, die Versuche $\mathrm{zu}$ wiederholen. Ich excidirte syphilitische Papeln von acht verschiedenen Patienten, die Queksilber in verschiedenster Quantität erhalten hatten (näInlich nach 2 Injectionen, nach 6 Injectionen und nach 12 Injectionen von je $0.1 \mathrm{Hg}$. salicylicum) und bei denen eine locale Quecksilberbehandlung der syphilitischen Papeln peinlichst vermieden war.

Diese excidirten Stückchen wurden genau nach J u st u s' Vorschrift zunächst in eine $14 \% \mathrm{ZnCl}_{2}$ (Zinkchlorid) Lösung 
bezw. in eine $11 \cdot 7 \% \mathrm{ZnSO}_{4}$ (Zinksulfat) Lösung gethan. Da nur ungenau angegeben war, wie lange die Stücke in der Flüssigkeit bleiben sollten - (J. schreibt „einige Tage") so habe ich einige Stïckchen $1 \mathrm{Tag}$, andere 2, resp. 4 Tage in der Flüssigkeit liegen lassen. Aus dieser Lösung brachte ich sie in Schwefelhydrogenwasser (gesättigte Schwefelwasserstofflösung), durch welches mehrere Stunden lang $\mathrm{H}_{4 \mathrm{~S}} \mathrm{~S}$ geleitet wurde. Hierauf stufenweise stärker werdender Alkohol, Paraffineinbettung und mikroskopische Untersuchung. Schon in a $\mathrm{k} \mathrm{r} \mathrm{o-}$ $\mathrm{skopiseh}$ war nach der $\mathrm{H}_{2} \mathrm{~S}$-Procedur ein mehr oder weniger bräunlicher Farbenton an den Stiickchen sichtbar, welcher indessen mit dem grösseren oder geringeren $\mathrm{Hg}$-Gehalt derselben ursächlich nicht in Zusammenhang gebracht werden konnte. Mikroskopisch waren in sämmtlichen Papeln schollige Massen von bräunlicher Farbe erkennbar. Thre Menge wechselte in den verschiedenen Präparaten. Am Rand des Schnittes hatte diese Masse den Schnitt diffus gefärbt. Dies war speciell am Epithel deutlich sichtbar, wo die Masse nicht nur den einzelnen Epithelzellen aufgelagert war, sondern die Interspinalräume mit dieser Masse gleichsam ausgespritzt zu sein schienen, so dass die Spalträume ganz damit erfüllt waren. Nach der Mitte des Schnittes zu war die beschriebene Masse nicht diffus, wie über den Schnitt ausgegossen, sondern in einzelnen unregelmässig geformten und unregelmässig angeordneten Theilchen von verschiedenster Grösse angeordnet. Je mehr nach der Mitte des Schnittes, desto weniger war von ihnen sichtbar. Ihre äussere Form und Begrenzung hielt sich da, wo sie klümpchenweise angeordnet war, keineswegs an die Zellform der Gegend, wo sie vorkam; auch war ein Zusammenhang mit den Gefässen nicht erkennbar. Oft waren die Klümpchen weit unter Zellgrösse, oft bedeutend grösser. Eine Aehnlichkeit mit der Zellform war nur im geschichteten Bindegewebe erkennbar; hier waren die Klümpchen oft spindelförmig, ähnlich den dort vorhandenen Zellen, entsprechend der Richtung der Bindegewebsbündel. Ein Zusammenhang mit Zellen war auch hier nicht ersichtlich.

Diese besonders starke Anbäufung der braunen Massen würde ganz dem in Fig. 3, Taf. VI. von $J$ u s t u s abgebildeten 
Schnitte entsprechen, welcher in der Randgegend viel stärker gebräunt erscheint als im Centrum.

Um nun einen specifischen Einflus der ron Justus beschriebenen und im Vorstehenden von mir z. T. bestätigten Befunde a uf das syphilitis che Gewebe annehmen zu können, wäre es nöthig, das Verhalten anderer, $\mathrm{ni} \mathbf{c h t} \mathrm{syphilitis} \mathrm{cher}$, aber ebenfalls mit Hg behandelter Hautaffectionen zum Vergleiche heranzuziehen. Da mir geeignetes menschliches Material nicht zur Verfügung stand, suchte ich die vorliegende Frage durch den Thierversuch zu entscheiden. 1ch hatte Gelegenheit, Organstückchen (Niere und Leber) von einem mit $\mathrm{Hg}$ behandelten Pferde zu untersuchen. Dieselben zeigten nun nach der oben beschriebenen Behandlung mit $\mathrm{ZnCl}_{2}$ und $\mathrm{H}_{2} \mathrm{~S}$ e be nfalls die geschilderten bräunlichen Klümpchen in derselben Form und Anordnung d. h. auch hier waren die unregelmässig zerstreuten Schollen in der Peripherie der Schnitte am stärksten angehäuft und nahmen gegen die Mitte zu ab.

Wir können nun hiernach nicht annehmen, dass die beschriebenen bräunlichen Massen einen specifischen Einfluss des Quecksilbers auf syphilitisches Gewebe beweisen, da auch im nichtsyphilitischen, aber Hg-haltigen Gewebe derselbe mikroskopische Befund erhoben werden konnte.

Es musste nunmehr festgestellt werden, in welchem $\mathrm{Zu}$ sammenhang die geschilderten bräunlichen Massen zum Quecksilbergehalt der Gewebe standen, ob insbesondere die Justus'sche Methode eine irgendwie quantitative Bestimmung des Hg-Gehaltes der Gewebe zuliess.

Ich injicirte zu diesem Zweck mehreren Kaninchen Sublimatlösungen von verschiedenster Concentration. Kleine Stückchen von Niere und Leber der Thiere wurden dann mit $\mathrm{ZnCl}_{2}$ und $\mathrm{H}_{2} \mathrm{~S}$ behandelt und mikroskopisch untersucht. Der grösste Theil der Nieren und Lebern wurde $\mathrm{che} \mathrm{m}$ is $\mathrm{ch}$ auf den $\mathrm{Hg}$-Gehalt geprüft d. b. die Organe wurden mit Kali chloricum und Salzsäure versetzt bis zur vollständigen Zerstörung der organischen Substanz gekocht, die restirende Flüssigkeit mit Kupferblechen behandelt und so der $\mathrm{Hg}$-Gehalt quantitativ festgestellt. Hierbei stellte sich heraus, wie es ja 
auch zu erwarten war, dass der Quecksilbergehalt der Nieren und Lebern der Menge des einverleibten $\mathrm{Hg}$ proportional war.

Was die mit $\mathrm{ZnCl}_{2}$ und $\mathrm{H}_{2} \mathrm{~S}$ behandelten Stücke anbetrifft, so konnte an den mikroskopischen Schnitten im Grossen und Ganzen der gleiche Befund erhoben werden, wie wir ihn oben bei den syphilitischen Papeln ausführlich beschrieben haben. Dass indessen die Menge der bräunlichen Klümpchen dem g rös seren oder geringeren Hg-Gehalt der thierischen Gewebe proportional war, war absolut nicht $z u$ constatiren. Im Gegentheil zeigten einige Organstückchen eines nur ganz schwach mit $\mathrm{Hg}$ behandelten Kaninchens, in denen auch chemisch nur wenig $\mathrm{Hg}$ nachgewiesen werden konnte, auffallend viel der erwähnten Schollen. Besonders an der Peripherie der Schnitte, entsprechend der Oberfläche der Stückchen, war das ganze Gewebe durch die bräunlichen Massen diffus verfärbt.

Dieses Missverhältniss zwischen dem chemisch festgestellten Quecksilbergehalt der Gewebe und der Menge der mikroskopisch nachweisbaren bräunlichen Klümpchen liess uns nunmehr zweifeln, ob die letzteren überhaupt mit dem in den Geweben befindlichen $\mathrm{Hg}$ in Beziehung gebracht werden konnten.

Es musste der Einwand ausgeschlossen werden, dass vielleicht durch die Behandlung mit den chemischen Agentien $\left(\mathrm{ZnCl}_{2}\right.$ und $\left.\mathrm{H}_{2} \mathrm{~S}\right)$ die bräunlichen Massen erst hineingebracht worden waren, quasi als Niederschläge von der $\mathrm{ZnCl}_{2}$ und $\mathrm{H}_{\mathrm{g}} \mathrm{S}-\mathrm{Behandlung}$ herrührten. Zu dem Zwecke war es nöthig, Controlversuche an $\mathrm{Hg}$ frei en Organstückchen anzustellen. Schon beim ersten Durchlesen der Justus'schen Arbeit fiel es uns auf, dass von derartigen Controlversuchen nichts erwähnt war.

Wir setzten also kleine Nieren- und Leberstücke von sicher $\mathrm{Hg}$-freien Kaninchen der $\mathrm{ZnCl}_{2}$ und $\mathrm{H}_{2} \mathrm{~S}$ Einwirkung wie bei den vorstehenden Versuchen aus. Auch hatte ich Gelegenheit, einige nicht syphilitische Papeln von sicher $\mathrm{Hg}$-freien Patienten und etliche syphilitische Papeln von noch nie mit $\mathrm{Hg}$ behandelten Patienten derselben Procedur unterwerfen zu können. $\mathrm{Zu}$ unserer grossen Ueberraschung entdeckten wir 
auch hier im mikroskopischen Bilde dieschon so oft erwähnten bräunlichen scholligen Gebilde. Da also auch in nicht mit $\mathrm{Hg}$ behandelten und sicher $\mathrm{Hg}$ freien Geweben nach Behandlung mit $\mathrm{ZnCl}_{2}$ und $\mathrm{H}_{2} \mathrm{~S}$ die beschriebenen Gebilde in mikroskopischen Schnitten sichtbar sind, können wir dieselben nicht mit der Quecksilberanwesenheit in Geweben ursächlich in Beziehung bringen. Ja, wir werden vielmehr zu der Annahme gedrängt, dass die bräunlichen, scholligen Massen als Niederschläge ron der $\mathrm{ZnCl}_{2}$ und $\mathrm{H}_{\mathbf{q}} \mathrm{S}$ Behandlung her zu betrachten sind oder aber physiologische Gebilde, die durch die $\mathrm{ZnCl}_{2}$ und $\mathrm{H}_{2} \mathrm{~S}$-Behandlung verändert sind, darstellen.

Es haben sich also nach dem Ergebniss meiner Untersuchungen die Hoffnungen nicht erfüllt, die wir auf die von $J$ ustu s beschriebene Methode gesetzt hatten. Da wir indessen hören, dass Justus weitere Untersuchungen über die Action des Quecksilbers auf das syphilitische Gewebe anstellen will, so möchten wir durch den negativen Ausfall unserer Nachprüfungen die vorliegende Frage noch nicht für abgeschlossen erachten, sondern einer weiteren, ausführlichen Darlegung von Seiten des genannten Autors entgegensehen. 